\title{
La cuantificación nominal indefinida desde una perspectiva diacrónica*
}

\author{
Indefinite nominal quantification from a \\ diachronic approach
}

María Mare**

\section{RESUMEN}

En el español de los siglos XIII y XIV es posible encontrar concorPalabras clave: dancia entre el cuantificador y el nombre, junto con el ítem de en una misma construcción cuantificada: muchos de árvoles [1275, Alfonso X, General Estoria]. En este trabajo recuperamos algunos criterios para identificar muchas de estas secuencias como ejemplos de cuantificación indefinida y así trazar un panorama cuantificación indefinida, partitividad, variación, español medieval. de las posibilidades de marcación entre el nombre cuantificado y el cuantificador.

\section{ABSTRACT}

In 13th and 14th-Century Spanish, it is possible to find agreeKeywords: ment between the quantifier and the noun, along with the item Indefinite de in the same quantified construction: muchos de árvoles [1275, quantification, Alfonso X, General Estoria]. In this paper, we revise some critePartitivity, ria that allow us to identify these sequences as examples of indefinite quantification and draw a picture of the possibilities of Variation, Old Spanish. marking between the quantified noun and the quantifier.

\footnotetext{
* Esta investigación es parte del proyecto de investigación "Adquisición, cambio y contacto lingüístico. Aspectos teóricos, descriptivos y pedagógicos" (04/J030). Universidad Nacional del Comahue.

** Argentina. Doctora en Ciencias del Lenguaje. Académica de la Universidad Nacional del Comahue/IPEHCS-Conicet, Neuquén, Argentina. maria.mare@fahu.uncoma. edu.ar.
} 


\section{Introducción al problema}

Desde la perspectiva de la lógica y de la semántica filosófica, la operación de cuantificar se entiende como "la acción de establecer cierta relación entre dos conjuntos” (Real Academia Española, RAE y Asociación de Academias de la Lengua Española, ASALE, 2009, p. 1377). Entre las posibilidades de cuantificación nominal que ofrece el español se reconoce aquella que distingue entre conjuntos definidos y conjuntos indefinidos. En las variedades estándar actuales es posible identificar dos patrones básicos en relación con la cuantificación indefinida: un cuantificador que concuerda en número y género con el nombre cuantificado (muchas personas); un cuantificador que presenta sus propios rasgos de número y persona y un nombre cuantificado que aparece precedido por de (un montón de personas). La combinación de ambas características (concordancia y presencia de $d e$ ) encabeza normalmente sintagmas determinantes definidos y da lugar a lo que se conoce como construcciones partitivas (muchas de las personas). Sin embargo, en el período que va desde el siglo XII al siglo XV, la distribución apuntada no parece ser tan clara y, tal como señala Camus Bergareche (2009), entre otros, es posible encontrar construcciones como las de (1). La bibliografía en general las clasifica como partitivas.

(1) a. con muchos de árvoles [1275, Alfonso X, General Estoria]

b. mucho de ganado [1400, Anónimo, Biblia Ladina]

En estos ejemplos, se observa tanto la presencia de de como de la concordancia entre el cuantificador y el nombre cuantificado, sin embargo, no resulta del todo claro si se trata de construcciones partitivas, es decir, una parte de un conjunto definido, o bien, de construcciones cuantificadas indefinidas. Cualquiera de las dos posibilidades supone una diferencia en relación con las formas en las que se materializan estos tipos de cuantificación en español actual, ya que como construcciones partitivas, carecen del determinante definido y como cuantificación indefinida tendríamos una marcación redundante o, en otros términos, no se esperaría la presencia de $d e$.

El objetivo de este trabajo fue establecer de la manera más clara posible los criterios para determinar qué tipo de cuantificación se produce en casos como los de (1), a partir de las distinciones desarrolladas en Falco y Zamparelli (2019). Una vez establecidos estos criterios, 
procuramos presentar una sistematización que contemplara la distribución de los marcadores gramaticales involucrados, a saber, la concordancia entre el cuantificador y el nombre cuantificado y la marcación diferencial del nombre cuantificado (con de en español o con caso genitivo en latín).

El artículo se organiza de la siguiente manera. En la sección que sigue presentamos una primera aproximación a los datos, a fin de revisar las nociones de partitividad y cuantificación indefinida. En la tercera parte recuperamos las discusiones que se han realizado en torno a la cuantificación nominal en latín, ya que las observaciones respecto de esta lengua podrían arrojar luz sobre los datos de (1). Como veremos en la cuarta parte, los criterios que reconocen Devine y Stephens (2006) son de utilidad para conformar y describir nuestro corpus. Finalmente, se explicitan algunas consideraciones acerca de los fenómenos de variación.

\section{Partitividad y cuantificación indefinida}

Como se mencionó en la introducción, las construcciones de (1) se clasifican como partitivas en la literatura descriptiva. Camus Bergareche (2009), por ejemplo, destaca que “[e]n el español medieval y clásico todos los cuantificadores indefinidos no universales, y especialmente poco, se presentan esporádicamente encabezando como pronombres frases prepositivas con de de valor partitivo ${ }^{1 "}$ (p. 921). Por su parte Keniston $(1937, \$ 20.8)$ las vincula con los complementos verbales con de (dadnos del agua [Cid, 2798]) $)^{2}$ y señala que ambas formas se abandonaron en el tránsito al español moderno. Observemos los ejemplos en (2), recuperados de Camus Bergareche (2009, pp. 921-922).

(2) a. hlegó muchos de povres quantos podió hallar [DN, 1250, 4.7.95 $]^{3}$

b. ¿cómmo non puedo sofrir unos pocos de días que biva en religión et sufra un poco de desabor? [Calila, 116]

1 Subrayado nuestro.

2 Ver López Palma (2010) para un estudio de estas construcciones a las que la autora denomina partitivas indefinidas.

3 González Ollé (1993). 
c. Et después que gelo ovo fecho muchas de vezes [Calila, 143]

d. que en poco de ora desenbargó la gente [ $D N, 1435,8.18 .319]$

e. assaz hallará de apassionados [apud Keniston 1937, §13.1]

f. açíaseme arto de mal [apud Keniston 1937, §13.1]

g. Y tienen bastante de comida [Poma, Nueva corónica, ca. 15951615, CORDE]

h. demasiado de comedimiento os hago en pedíroslo [Avellaneda, Don Quijote de la Mancha, 1614, CORDE]

i. çercarle pueden por ventura tantos de perdimientos, que non se podrá dellos anparar [Calila, 345]

Independientemente del hecho de que aparezca la marca de, típicamente asociada a la partitividad, entendemos que algunas de estas secuencias podrían no tener una lectura partitiva (por ejemplo, $m u$ chos de los árboles en la), sino que serían casos de cuantificación indefinida (muchos árboles en 1a). De acuerdo con la bibliografía (Brucart 1997; Falco \& Zamparelli 2019; Martí-Girbau 2010, y las referencias allí citadas), la construcción partitiva es una frase nominal que se utiliza para referir a un subconjunto o a una subparte de otro referente -el antecedente (coda)—, que ha sido introducido previamente en el discurso, propiedad que típicamente se manifiesta a través de mecanismos sintácticos formales (uso de pronombres posesivos, determinantes definidos, relativas restrictivas, etc.). Esta característica - la de presentar una coda definida o, por lo menos, referencial-, se conoce como "partitive constraint" (restricción partitiva) (Jackendoff 1977, p. $113)^{4}$. En cambio, la cuantificación indefinida implica la cuantificación de un nombre sin referencia a un conjunto definido.

En la bibliografía generativista esta distinción se formaliza a partir de la diferencia entre sintagmas nominales (SN) y sintagmas determinantes (SD). El SD puede contener un SN, pero se trata de una frase más compleja, con proyecciones que codifican sus propiedades refe-

4 Jackendoff (1977) define a esta restricción de la siguiente manera: "In an of-N"' construction interpreted as a partitive, the $\mathrm{N}$ "' must have a demonstrative or genitive specifier"' (p.113). En español: Si una construcción de-N"' es interpretada como partitiva, el N"' debe tener un especificador demostrativo o genitivo.' 
renciales (Abney, 1987). En este sentido, en las secuencias a continuación, el cuantificador indefinido muchos se vincularía con un SN en (3a) y con un SD en (3b), dando lugar a la lectura cuantitativa indefinida y partitiva, respectivamente.

(3) a. Muchos [sN docentes] vinieron a la reunión [Cuantificación indefinida]

b. Muchos de [sD los docentes] vinieron a la reunión [Construcción partitiva]

Ahora bien, para el período que estamos estudiando, Suñer, Roca, Pujol, Castillo y Batllori (2002) destacan usos de cuantificadores indefinidos que preceden a un nombre con determinante definido y arrojan lecturas partitivas. Los ejemplos de (4) muestran que esa relación de partitividad se establecería independientemente de la presencia del ítem $d e$, a diferencia de lo que se observa en (3a) para el español estándar actual.

(4) a. algunos nuestros arrendadores e rrecabdadores mayores

[Anónimo, Cuaderno de leyes, S XV, CORDE]

b. Y assí embió a otros muchos sus amigos, aliados y servidores

[Rodríguez de Montalvo, Amadís de Gaula, 1482-1492, CORDE]

c. pero aun de algunas sus particularidades salían delectables fontezillas de filosophia

[Celestina: 070.3 $]^{5}$

Para agregar una dificultad más al análisis de datos como los de (1) y (2), resulta fundamental señalar que dentro de la cuantificación indefinida, Brucart (1997) reconoce para el español una construcción que aparece en otras lenguas y se denomina pseudo-partitiva, dado que manifiesta el mismo marcador que se halla en las partitivas, i.e., el ítem de y que Bello (1847/1988) ya había referido previamente de igual modo. Como señalan Falco y Zamparelli (2019), estas secuencias presentan un elemento nominal semifuncional (montón, parva,

5 Los ejemplos de (4) son tomados de Suñer, Roca, Pujol, Castillo y Batllori (2002). 
mogollón $)^{6}$ y un nombre desnudo, y el referente no está ligado a un antecedente previamente establecido.

(5) Un montón de docentes vinieron a la reunión. [construcción pseudo-partitiva]

Ahora bien, en los ejemplos de (1) y (2), que Camus Bergareche (2009) describe como partitivos, encontramos secuencias que sí son claramente partitivas, como (2a) y (2b), en las que la presencia de cláusulas relativas restrictivas desencadena la lectura definida del conjunto cuantificado. Sin embargo, otros casos podrían considerarse de manera similar a las construcciones pseudo-partitivas, es decir, como cuantificaciones indefinidas. Asimismo, los ejemplos de (4) muestran que no solo la presencia del artículo definido está parcialmente estabilizada, sino que también el ítem de puede omitirse en las construcciones partitivas.

Con todo, resulta útil recuperar las definiciones específicas, ya que la diferencia entre ambas construcciones en español actual proviene en gran medida de la presencia del ítem de y de las marcas de definitud en el nombre cuantificado. Precisamente, estas dos características presentan un comportamiento inestable en el período estudiado, como indican Suñer, Roca, Pujol, Castillo y Batllori (2002) para de y Company Company (1991) para el artículo definido?

\section{Las estrategias de cuantificación nominal en latín clásico}

En este apartado nos interesa revisar las estrategias de cuantificación nominal del latín clásico, ya que esta lengua presenta algunas particularidades que pueden resultar iluminadoras para avanzar en la

6 Los objetos sintácticos que se inscriben bajo el rótulo de cuantificadores suponen una relación de predicación con el elemento cuantificado (en consonancia con MartíGirbau, 2010). Sin embargo, no todo lo que se denomina "cuantificador" tiene la misma estructura interna (en consonancia con las diferencias que muestran Di Tullio y Kornfeld, 2012). Ver también Sánchez López (1999).

7 Company Company (1991) distingue tres etapas en el avance del artículo a las diversas clases de nombres. Primera etapa: hasta fines del siglo XIII $\rightarrow$ nombres concretos, sustantivos animados y sustantivos genéricos en función de sujeto. Segunda etapa: hacia mediados del siglo XIV $\rightarrow$ genéricos no humanos e ítems de referencia única. Tercera etapa: siglo XV $\rightarrow$ el artículo se emplea con menos restricciones y se presenta con sustantivos abstractos y de masa. 
descripción de los datos que exponemos en la sección siguiente, en relación con las variedades actuales del español. Como es sabido, el latín clásico se caracteriza por la ausencia del artículo definido, lo que no implica la carencia del contraste SD/SN que recuperamos en el apartado anterior (Ledgeway, 2012). Es decir, la innovación de las lenguas romances respecto del latín no sería el resultado de pasar de una lengua con SNs a lenguas con SDs, sino debido a la manera en la que se distribuye la información gramatical dentro del SD, cuando se trata de expresiones referenciales. En este sentido, el cambio que acompaña la emergencia del artículo definido es la pérdida del sistema de casos, como ha sido señalado ampliamente en la bibliografía (Lapesa, 2000).

En cuanto a la cuantificación nominal en latín, Spevak (2014) reconoce dos tipos de cuantificadores según la manera en la que se relacionan con el nombre cuantificado. Así, distingue los adjetivales, que presentan concordancia (6) y los nominales, en los que el nombre cuantificado está marcado con caso genitivo y el cuantificador presenta información de género y número en sí mismo (7)

(6) Cuantificadores "adjetivales"

a. equi multi [Sal. Jug. 29.6]

'muchos caballos'

b. pauci milites [Caes. Civ. 2.44.1]

'pocos soldados'

c. nonnulli centuriones [Caes. Civ. 1.80.5]

'algunos centuriones'

d. aliquot fontes [Sal. Jug. 89.6]

'varias fuentes'

(7) Cuantificadores "nominales" + genitivo

a. magnam turbam ignotorum deorum [Cic. N.D. 1.39]

'una enorme turba de dioses desconocidos'

8 Los ejemplos han sido tomados de Spevak (2014, pp. 49-50). Las traducciones al español son propias. 
b. cum grege praedonum [Cic. Dom. 24]

'con un montón de ladrones'

c. cum magna caterva togatorum [Cic. S. Rosc. 135]

'con una caterva de ciudadanos en toga'

d. Magnum numerum frumenti [Cic. Verr. 2. 176]

'gran cantidad de maíz'

Las construcciones como las de (7) han sido incluidas muchas veces bajo el rótulo de genitivos partitivos, junto con secuencias como las de (8), en las que la frase marcada con caso genitivo está encabezada por un pronombre posesivo (indudablemente, un SD). Sin embargo, Kühner y Stegmann (1912) y Ernout y Thomas (1954) utilizan la denominación de genitivo de cantidad, dado que la relación semántica entre el cuantificador y el nombre cuantificado no es de parte-todo. De hecho, como señala Pinkster (2015) únicamente los ejemplos de (8) tienen como construcción alternativa sintagmas preposicionales encabezados por de y ex (9) (también Kühner y Stegmann, 1912). Como puede verse, los complementos de de y ex en (9) están precedidos por una expresión definida (destacada en cursiva a los fines expositivos).

(8) Cuantificadores "nominales" + genitivo con claro valor partitivo

a. partem suarum copiarum [Caes. Gal. 2.9.4]

'una parte de sus tropas'

b. plerique nostrorum oratorum [Cic. Orat. 143]

'la mayoría de nuestros oradores'

(9) Cuantificadores + nombre introducido por preposición con valor partitivo

a. Partis de nostris bonis [Ter. Hau. 652]

'parte de nuestras posesiones'

b. Ex illa pecunia magnam partem [Cic. Div. Caec. 57]

'una parte considerable de esta suma de dinero'

c. Pleraque de iis [Cic. De Orat. 2.107]

'La mayoría de estos' 
e. Nemo de nobis [Cic. Tusc. 5. 105]

'ninguno de nosotros'

Si bien estos datos parecerían indicar que existe un criterio claro para distinguir entre cuantificación indefinida y partitividad en latín, Devine y Stephens (2006) muestran un panorama más complejo al comparar dos construcciones con cuantificadores nominales en las que el nombre cuantificado presenta caso genitivo (pars y magnus numerus). Señalan que pars solo aparece con construcciones partitivas, mientras que magnus numerus aparece con construcciones cuantificadas indefinidas. A fin de diferenciar ambos valores, los autores siguen esta definición: la restricción partitiva (ver la sección 2) supone que el genitivo partitivo debe ser definido (o específico o indefinido restringido). Con magnus numerus, en cambio, los genitivos son típicamente indefinidos.

Archers $^{9}$ is a bare plural and $\operatorname{grain}^{10}$ is a bare mass noun, so this type of partitive genitive may belong to the class of pseudo partitives. It follows that while the post modifier genitives with pars were mostly presuppositional, those with magnus numerus are often new information (Devine \& Stephens, 2006, p. 375) ${ }^{11}$.

Estos autores proporcionan así dos criterios relevantes para distinguir construcciones partitivas de construcciones cuantificadas indefinidas cuando la presencia del artículo definido no constituye necesariamente un criterio: (1) los indefinidos restringidos que, como tales, tienen lectura partitiva, independientemente de la presencia de un ítem vinculado con la definitud y (2) el orden de constituyentes ${ }^{12}$. La noción de indefinición restrictiva, también mencionada en Falco y Zamparelli (2019), nos lleva a considerar el contexto en el que aparecen las secuencias de cuantificador $+d e+\mathrm{SN}$, a fin de determinar si

9 "Magnum numerum levis armaturae et sagittatiorum 'un gran número de tropas ligeramente armadas y de arqueros (Caes. BC 3.62), ejemplo (209)" (Devine \& Stephens, 2006, p. 374).

10 Frumenti en (7d).

11 Arqueros es un plural desnudo y maíz es un nombre de masa desnudo, por lo que este tipo de genitivo partitivo pertenecería a la clase de los pseudo-partitivos. Esto surge a partir del hecho de que mientras el posmodificador genitivo es más presuposicional con pars 'parte', aquellos que se combinan con magnus numerus 'un gran número' suponen generalmente información nueva. (Traducción nuestra).

12 Este segundo criterio es cuestionado por Spevak (2014). 
estamos ante una relación de parte-todo o ante la cuantificación de un conjunto indefinido.

\section{Observaciones respecto de los datos}

El período de tiempo estudiado va desde el siglo XII al siglo XV, es decir, lo que se conoce como español medieval (Eberenz, 2000; 2009; Lapesa, 1981; 2000; Menéndez Pidal, 1940/1999; Penny, 1991; Sánchez Lancis, 2009). Los datos analizados fueron extraídos fundamentalmente del Corpus Diacrónico del Español (CORDE). Para la selección de las obras a considerar, se tuvieron en cuenta los criterios establecidos en Rodríguez Molina y Octavio de Toledo y Huerta (2017) respecto de la lingüística de corpus y, en especial, de la distinción que hacen entre textos y testimonios. A tal fin, los textos de los cuales hemos extraído los datos han sido seleccionados a partir de la herramienta conocida como Cordemáforo, que apunta a garantizar la fiabilidad filológica de los materiales del CORDE. Dicha fiabilidad se obtiene, por un lado, de la calidad filológica de los testimonios que sirven de base a los textos del CORDE, es decir, si son originales, copias cercanas al original o copias tardías y, por otro lado, el tipo de acceso al texto que se proporciona, a saber, transcripción paleográfica, edición crítica, etc.

En cuanto a los datos, siguiendo el criterio empleado en trabajos anteriores (Pérez Guarino y Mare, 2016) se delimitó el conjunto de cuantificadores indefinidos y el tipo de construcción. Los cuantificadores que se estudiaron son algun-, as(s)az, bastante, demasiad-, fart-/hart-, feten, much-, ningun-, poc-, vari-, con todas las posibilidades de flexión en género y número en los casos que lo permitieran. Algunos de estos ítems cuantifican adjetivos y, si bien esta combinación no forma parte del presente artículo, es pertinente destacar que relevamos casos de cuantificador $+d e+$ adjetivo, con lo que la fluctuación que mostramos para la cuantificación nominal parece extenderse también al ámbito adjetival. Dejamos estos casos para futuras investigaciones.

El segundo criterio fue la delimitación del tipo de construcción. Dejando al margen las consideraciones que expondremos más adelante, nos enfocamos aquí en las secuencias de cuantificador $+d e+\mathrm{SN}$, es decir, nombre sin determinante y elementos no pronominales, entendiendo que los pronombres son SDs (Abney, 1987; Panagiotidis, 2002). 
En este sentido, quedaron fuera de la investigación ejemplos como los de (4) y datos como el de (10), que constituyen estructuras partitivas en el sentido expuesto en la segunda parte de este artículo. También procuramos descartar los indefinidos restringidos en línea con lo apuntado por Devine y Stephens (2006) para el latín, es decir, aquellas secuencias que si bien presentan la forma cuantificador $+d e+\mathrm{SN}$, tienen un antecedente definido en el discurso, como en (11).

(10) Et si a alguno dellos la sentencia non ploguiere [Anónimo, Fuero de Zorita de los Canes, 1218- 1250, CORDE]

(11) a. Quando en la mañana salién a los lavores,/dieron salto en ellos essos cavalgadores,/mataron e prendieron muchos de labradores,/de quanto lis fallaron non fueron más señores

[Berceo, Vida de Santo Domingo de Silos, 1236, CORDE]

b. Porque vimos ya muchos de juyzes \& muchos de sayones que por cobdicia passaban el mandado de la Ley

[A., Fuero juzgo, 1250- 1260, CORDE]

Los ejemplos con los distintos cuantificadores indefinidos seleccionados varían en términos de productividad dentro del corpus y de las construcciones en las que pueden aparecer. Alguno, mucho, poco y tanto, con todas las posibilidades flexivas, son los que más ejemplos presentan en el corpus y se encuentran tanto en concordancia con un nombre precedido por $d e$ (QCONC $+d e+\mathrm{SN}$, en adelante), como sin esta marcación (QCONC + SN, en adelante), además de hallarse en construcciones partitivas. $A s(s) a z$ 'ni poco ni mucho', tiene un valor que cubre actualmente bastante y modifica también verbos, adjetivo y adverbios. Como modificador del nombre, aparece con complemento preposicional, pero también antepuesto o pospuesto al sustantivo y no presenta flexión (Eberenz, 2000), por lo que la presencia del ítem de es esperable. También se registra un uso partitivo. Para ninguno se encuentran pocos ejemplos de Qconc $+d e+\mathrm{SN}$. Bastante se registra en el CORDE a partir de 1400, sin embargo, no encontramos datos de bastante + de + SN (Camus Bergareche, 2008). Asimismo, consideramos los cuantificadores demasiado, farto/harto, femen y varios, pero no pudimos encontrar ejemplos relevantes, es decir, en estructuras de QCoNC + de + SN. A continuación presentamos algunos de los ejem- 
plos de cuantificación indefinida que relevamos siguiendo los criterios planteados en las secciones precedentes.

(12) Algun-13

a. con algunas de guisas los ternién amansados

[A., Libro de Alexandre, 1240-1250, CORDE]

b. Et tod aquel que alguno de conceio defendiere

[A., Fuero de Zorita de los Canes, 1218-1250, CORDE]

c. será fecha querellando sse algunos de males o de dannos que rreçebieron de aquel ${ }^{14}$

[A., Espéculo de Alfonso X, 1260, CORDE]

(13) As(s)az

a. \& fizieron assaz de bien

[A., La gran conquista de Ultramar, 1293, CORDE]

b. otros signos cuntieron assaz de marabella

[Berceo, Loores de nuestra Señora, 1236-1246, CORDE]

c. ca yo he asaz de oro e de plata

[A., Traducción de Lanzarote del lago, 1414, CORDE]

d. e estando y asaz de gente

[A., Proceso judicial..., 1414, CORDE]

e. en este reino ay assaz de buenos cavalleros

[A., Colloquio de viejo, 1532, CORDE]

f. sabe que ha assaz de rocas alrededor

[Poza, Hydrografía, 1585, CORDE]

13 A partir del siglo XIV no se encuentra esta configuración en el corpus y la presencia de de es concomitante con un SD definido, dando lugar de manera no ambigua a la interpretación partitiva.

14 Este ejemplo podría tener valor partitivo. En el mismo texto aparece sistemáticamente un SD definido: algunos de los pressos/los adelantados/los otros/aquellos. 
(14) Much-

a. que les fará mucho dalgo y mucho de plazer si foren obedientes [A., Poridat de Poridades, 1250, CORDE]

b. \& echan della una poca en un vaso \& mucha de agua [Alfonso X, Lapidario, 1250, CORDE]

c. en la mesura veras muchos de bienes \& muchas bondades. En la mesura veras muchas de maldades

[A., Castigos BNM ms. 6559, 1293, CORDE]

d. iacemos en grand culpa por muchas de razones

[Berceo, Vida de Santo Domingo de Silos, 1236, CORDE]

(15) Poc-

a. sanó Sancto Domingo en pocas de jornadas

[Berceo, Vida de Santo Domingo, 1236, CORDE]

b. y que no coman más, sinon un poco de pan y una poca de agua [A., Fuero juzgo, 1250-1260, CORDE]

c. vio una tinaja en que avía un poco de trigo

[A., Calila e Dimna, 1251, CORDE]

d. E a pocos de meses murió

[Alfonso X, Estoria de Espanna, 1270, CORDE]

e. fasta el rio do a unas pocas de figueras

[A., Repartimiento de Murcia, 1257- 1271, CORDE]

(16) Ningun-

a. Muchos lugares en que non an ninguna de agua \& de pluvia

[A., Fuero juzgo, 1250- 1260, CORDE]

b. otrossi non caya ninguno de plazo

[A. Fuero de Béjar, 1290-1293, CORDE] 
(17) Tant-

a. es tanto de amor que puso Dios

[A., Calila e Dimna, 1251, CORDE

b. \& diole tantos de golpes con aquella maça

[A., Libro del cavallero Cifar, 1300-1305, CORDE

c. E este fizo tantas de buenas cosas

[A., Crónica del Moro Rassi, 1300- 1344, CORDE]

Como puede observarse, la mayoría de los datos pertenecen al siglo XIII y de los cuantificadores que aparecen con más frecuencia en el corpus (alguno, mucho, poco y tanto), solo pudimos identificar ejemplos pertinentes para el siglo XIV con tant-. Llama la atención la ausencia de datos relevantes con el cuantificador poc- dado que la estructura $p o c-+d e+\mathrm{SN}_{\mathrm{CONC}}$, con valor indefinido, es la que se reconoce en muchas variedades actuales y la que tiene una presencia importante en el Corpus diacrónico y diatópico del español de América (CORDIAM), en el que no pudimos encontrar, sin embargo, esta secuencia con otros cuantificadores. Por su parte, el cuantificador $a s(s) a z$, que se ha perdido en la lengua en general, aparece en el CORDE con ejemplos que corresponden a diferentes siglos y convive con estructuras cuantificadas en las que no aparece de y también con construcciones con interpretación partitiva.

Con todo, entendemos que los datos de (12) a (17) constituyen ejemplos de cuantificación indefinida y muestran que, al menos durante el siglo XIII, conviven tres maneras de establecer la relación entre el cuantificador y el nombre cuantificado, dos de las cuales (las primeras), son las que finalmente se identifican en distribución complementaria en las variedades estándar del español actual. Las diferencias entre estos tres esquemas parecen ser estrictamente formales y no el resultado de modificaciones vinculadas con aspectos estilísticos o de estructura informativa.

(18) Estructuras de cuantificación indefinida

a. $\mathrm{Q}+d e+\mathrm{SN}$ (un gran número de razones)

b. QCONC + SN (muchas razones) 


\section{c. QCONC $+d e+\mathrm{SN}$ (muchas de razones)}

Cabe mencionar que la evidencia empírica que permite identificar estructuras como la de (18c) con lectura indefinida no es numerosa en el corpus y está restringida, fundamentalmente, a los siglos XIII y XIV. Sin embargo, los datos muestran un tipo de alternancia que no resulta ajena a otros procesos que es posible identificar en las variedades del español, específicamente, en términos de doble marcación de información gramatical, como se comentará en el próximo apartado.

\section{Observaciones finales}

En este recorrido, hemos procurado revisar las secuencias del español medieval que presentan la estructura QCONC $+d e+\mathrm{SN}$, a fin de identificar criterios que nos permitan evaluar si se trata de construcciones indefinidas o partitivas.

A partir de las distinciones en la bibliografía, pudimos diferenciar aquellos casos en los que el contexto permite recuperar un antecedente, lo que se conocen como indefinidos restrictivos, que tienen lectura partitiva. También de acuerdo con la restricción partitiva descartamos todas aquellas secuencias en las que el nombre cuantificado está en relación con un ítem que lo determina. Los datos del latín muestran que este dominio está sujeto a alternancias también en esta lengua y que resulta necesaria la búsqueda de criterios que no dependan estrictamente de la presencia o ausencia del artículo definido para distinguir entre construcciones partitivas y de cuantificación indefinida.

Con todo, vemos que la concordancia entre el cuantificador y el nombre cuantificado y la marcación diferencial del nombre cuantificado (con de o con caso genitivo) están en distribución complementaria en el español actual y en latín, cuando de cuantificación indefinida se trata. En el español del siglo XIII, ambas formas conviven con una tercera opción que parece una combinación de ambas, lo que identificamos como $\mathrm{Q} \mathrm{CONC}+d e+\mathrm{SN}^{15}$. En principio, entonces, po-

15 Cabe mencionar que si bien no hemos realizado un trabajo estadístico, ya que entendemos que ello escapa a los fines de nuestro recorrido, se desprende del trabajo de búsqueda de datos que esta tercera opción es mucho menos productiva que las dos que se conservan en español actual, al menos a partir de los que surge en el CORDE. 
dría pensarse que en español actual y en latín, cuando la concordancia no tiene lugar - porque tanto el cuantificador como el cuantificado presentan sus propios rasgos de número y género-, la operación que aplica es la marcación diferencial (ítem de o caso genitivo). Lo que parecería suceder en la gramática del español de este periodo, en cambio, es la posibilidad de una doble marcación (con concordancia y marcación diferencial). La doble aplicación de mecanismos que vinculan dos objetos sintácticos en un ámbito particular no es ajena a la variación lingüística, ya que se observa en estructuras posesivas (su casa de Juan), de concordancia verbal (diganselon) e incluso de concordancia con marcación diferencial de objeto (se detuvieron a los delincuentes), en la que la relación entre el verbo y el SD los delincuentes se establece en términos de concordancia, pero también de marcación con $a$.

Si bien cada uno de estos temas merece una presentación detallada, que excede el alcance de este trabajo, lo que queremos señalar aquí es que parece claro que ciertas distribuciones funcionan de manera diferente en las distintas variedades de las lenguas, dando lugar a marcaciones redundantes. En línea con la hipótesis de la uniformidad (Roberts, 2007; Roberts \& Roussou, 2003), que postula que las lenguas del pasado no son esencialmente diferentes de las del presente, en cuanto que presentarían la misma gramática universal y los mismos mecanismos, no es de extrañar que en un recorrido diacrónico encontremos una variación similar.

\section{Referencias bibliográficas}

Abney, S. (1987). The English noun phrase in its sentential aspect (Tesis de doctorado). Massachusetts Institute of Technology, MIT, Massachusetts.

Academia Mexicana de la Lengua, CORDIAM. (s/f). Corpus diacrónico y diatópico del español de América. Recuperado de http:// www.cordiam.org/

Bello, A. (1988). Gramática de la lengua castellana: destinada al uso de los americanos. Madrid: Arco Libros. (Trabajo original publicado en 1847).

Brucart, J. M. (1997) Concordancia ad sensum y partitividad en español. En M. Almeida y J. Dorta (Eds.), Contribuciones al estu- 
dio de la lingüistica hispánica. Homenaje al profesor Ramón Trujillo. Vol. I (pp. 157-184). Tenerife: Montesinos.

Camus Bergareche, B. (2008). De adjetivos a cuantificadores: un fragmento de la historia de los indefinidos románicos. En. I. Olza Moreno, M. Casado Velarde, y R. González Ruiz (Coords.), Actas del XXXVII Simposio Internacional de la Sociedad Española de Lingüística (pp. 87-96). Pamplona: Universidad de Navarra.

Camus Bergareche, B. (2009). Cuantificadores I. Los cuantificadores propios. En C. Company Company (Dir.), Sintaxis histórica de la lengua española. Parte II: La frase nominal (pp. 881- 962). México, D.F.: Universidad Autónoma de México, UNAM-Fondo de Cultura Económica, FCE.

Company Company, C. (1991). La frase sustantiva en el español medieval. Cuatro cambios sintácticos. México, D.F.: Universidad Autónoma de México, UNAM-Instituto de Investigaciones Filológicas.

Devine, A. \& L. Stephens (2006). Latin word order, structured meaning and information, Oxford: Oxford University Press.

Di Tullio, A. y Kornfeld, L. (2012). Cuantificadores gramaticalizados del registro coloquial. En A. Di Tullio (Coord.), El español de la Argentina. Estudios gramaticales (pp. 105-127). Buenos Aires: Eudeba.

Eberenz, R. (2000). El español en el otoño de la Edad Media. Sobre el artículo y los pronombres. Madrid: Gredos.

Eberenz, R. (2009). La periodización de la historia morfosintáctica del español: propuestas y aportaciones recientes. Cahiers d'études Hispaniques Médiévales, 32(1), 181-201. https://doi. org/10.3406/cehm.2009.2072

Ernout, A. y F. Thomas. (1954). Syntaxe Latine. Paris: Klincksieck.

Falco, M. \& R. Zamparelli. (2019). Partitives and partitivity. Glossa: A Journal of General Linguistics, 4(1), 111, 1-49. http://doi. org/10.5334/gjgl.642

González Ollé, F. (1993). Lengua y literatura española medievales. Madrid: Alarcos libros.

Jackendoff, R. (1977) X'-Syntax. A study of phrase structure. Cambridge: Massachusetts Institute of Technology Press.

Keniston, H. (1937). The syntax of Castilian prose. The 16th Century. Chicago: Chicago University Press. 
Kühner, R. y C. Stegmann. (1912). Ausführliche Grammatik der lateinischen Sprache. Hannover: Hahn.Lapesa, R. (1981). Historia de la lengua española. Madrid: Gredos.

Lapesa, R. (2000). Estudios de morfosintaxis histórica del español. Madrid: Gredos.

Ledgeway, A. (2012). From Latin to romance: Morphosyntactic typology and change. Oxford: Oxford University Press.

López Palma, H. (2010). Las construcciones partitivas indefinidas en el español medieval. En P. Cano López, S. Cortiñas Ansoar, B. Dieste Quiroga, I. Fernández López, y L. Zas Varela (Eds.), Actas del XXXIX Simposio internacional de la Sociedad Española de Lingüistica (art. 22). Santiago de Compostela: Universidad de Santiago de Compostela.

Martí-Girbau, N. (2010). The Syntax of Partitives. Tesis doctoral. Universidad Autónoma de Barcelona.

Menéndez Pidal, R. (1999). Manual de gramática histórica del español (23 edición). Madrid: Espasa. (Trabajo original publicado en 1940).

Panagiotidis, P. (2002). Pronouns, clitics and empty nouns. Amsterdam: Benjamins.

Penny, R. (1991). Gramática histórica del español. Madrid: Ariel Letras.

Pérez Guarino, A. y Mare, M. (2016). Cambios en el sistema de cuantificación en español en torno a la ocurrencia del ítem de. En M. Fernández (presidencia), IV Congreso nacional. El conocimiento como espacio de encuentro. Simposio llevado a cabo en la Facultad de Lenguas, Universidad Nacional del Comahue, Argentina.

Pinkster, H. (2015). Oxford Latin syntax. Volume 1: The simple clause. Oxford: University Press.

Real Academia Española, RAE y Asociación de Academias de la Lengua Española, ASALE. (2009). Nueva gramática de la lengua española. Madrid: Espasa.

Real Academia Española, CORDE. (s/f). Banco de datos (en línea). Corpus diacrónico del español. Recuperado de http://www. raes.es

Roberts, I. \& A. Roussou (2003). Syntactic change: A minimalist approach to grammaticalization. Cambridge: Cambridge University Press. 
Roberts, I. (2007). Diachronic syntax. Oxford: Oxford University Press.

Rodríguez Molina, J. y Octavio de Toledo y Huerta, A. (2017). La imprescindible distinción entre texto y testimonio: el CORDE y los criterios de fiabilidad lingüística. Scriptum Digital, 6, 5-68. Recuperado de https://www.raco.cat/index.php/scriptumdigital/article/view/329258

Sánchez Lancis, C. (2009). Corpus diacrónicos y periodización del español. Cahiers d'études Hispaniques Médiévales, 32(1), 158180. https://doi.org/10.3406/cehm.2009.2071

Sánchez López, C. (1999) Los cuantificadores: clases de cuantificadores y estructuras cuantificativas. En I. Bosque y V. Demonte (Eds.), Gramática descriptiva de la lengua española, vol. I, (pp. 1025-1128). Madrid: Espasa.

Spevak, O. (2014). The noun phrase in classical Latin prose. Leiden: Brill.

Suñer, A., Roca, F., Pujol, I., Castillo, E., y Batllori, M. (2002). Alternancia de códigos en la expresión del caso partitivo. Recuperado de https://dugi-doc.udg.edu/handle/10256/3950 